\title{
Trastornos mentales entre personas con enfermedad cardiaca. Resultados del Estudio Nacional de Salud Mental, Colombia 2003
}

\author{
José Posada-Villa, MD¹, José Moreno² \\ ${ }^{1}$ Universidad Colegio Mayor de Cundinamarca, ${ }^{2}$ Universidad Nacional de Colombia \\ Correspondencia: hmontoyap@yahoo.com \\ Recibido: 07-18-07 / Aceptado: 08-15-07
}

\begin{abstract}
Resumen
Este artículo tiene como objetivos presentar la prevalencia estimada de trastornos del estado de ánimo, ansiedad y por consumo de alcohol en personas con enfermedad cardiaca en Colombia; e identificar los trastornos mentales específicos más fuertemente asociados con enfermedad cardiaca. Se aplico una encuesta de hogares en personas entre 18 y 65 años en Colombia $(n=4.426)$. Los trastornos mentales fueron evaluados con el WMH-CIDI, una entrevista diagnóstica estructurada. Los trastornos considerados en este estudio incluyen trastornos de ansiedad (trastorno de ansiedad generalizada, trastorno de angustia/agorafobia, trastorno por estrés post traumático y fobia social), trastornos del estado de ánimo (trastorno distímico y trastorno depresivo mayor) y trastornos por consumo de sustancias (abuso de alcohol y dependencia del alcohol). La enfermedad cardiaca fue determinada por auto reporte. El nivel de asociación entre estos trastornos y la enfermedad cardiaca fue evaluado usando razones de Odds ajustadas por edad y sexo.

Después de ajustar por edad y sexo, los trastornos específicos de ansiedad y del estado de ánimo fueron significativamente más prevalentes en personas con enfermedad cardiaca ( $\mathrm{p}>0.000$ ?). Los valores de los OR's ajustados $(95 \%$ intervalo de confianza) fueron $2.6(1.5,4.8)$ para trastorno depresivo mayor, $5.6(1.8,17.4)$ para trastorno distímico, $1.6(0.4,6.3)$ para trastorno de ansiedad generalizada, $2.5(1.0,6.3)$ para trastorno de angustia con agorafobia y $1.5(0.3,7.3)$ para abuso/dependencia del alcohol en personas con enfermedad cardiaca versus personas sin enfermedad cardiaca. Posterior al ajuste por edad y sexo, la prevalencia estimada de los trastornos del estado de ánimo y de ansiedad resulta mayor en personas con enfermedad cardiaca. La fuerza de la asociación de trastornos específicos del estado de ánimo y de ansiedad es consistente a través de estos trastornos.
\end{abstract}

Palabras claves: agorafobia, angustia, depresión, enfermedad cardiaca, fobia social.

\begin{abstract}
The objectives of this article are to present the estimated prevalence of mood, anxiety and alcohol abuse/ dependence disorders in people with heart disease in Colombia, and to identify the specific mental disorders more strongly associated with heart disease. A population survey of household-residing adults was carried out in Colombia.
\end{abstract}


Disorders considered in this study include anxiety disorders (generalized anxiety disorder, panic disorder/agoraphobia, posttraumatic stress disorder, and social phobia), mood disorders (dysthymia and major depressive disorder), and substance use disorders (alcohol abuse and dependence). Heart disease was ascertained by self-report. The association level between these disorders and the cardiac disease was evaluated using ODDS ratios adjusted by age and sex.

After adjusting for age and sex, specific mood and anxiety disorders were more prevalent in persons with heart disease. The estimates of the adjusted OR's $(95 \% \mathrm{CI})$ were $2.6(1.5,4.8)$ for major depressive disorder, $5.6(1.8$, $17.4)$ for dysthymia, $1.6(0.4,6.3)$ for generalized anxiety disorder, $2.5(1.0,6.3)$ for panic disorder/agoraphobia and $1.5(0.3,7.3)$ for alcohol abuse/dependence among people with versus people without heart disease. Later to the adjustment by age and sex, the estimated prevalence of the mood and anxiety disorders is bigger in persons with heart disease. The strength of association of specific mood and anxiety disorders with heart disease is consistent across disorders.

Key words: anxiety, agoraphobia, depression, heart disease, social phobia.

\section{Introducción}

La depresión y la enfermedad isquémica cardiaca son comunes en personas mayores y ambas son las principales causas de carga de la enfermedad a nivel mundial (1-3). La depresión tiene una gran carga de discapacidad en la población ya que ocurre generalmente en la primera mitad de la vida y con frecuencia tiene un curso crónico recurrente. Investigaciones previas en países desarrollados han encontrado que las personas con enfermedad cardiaca tienen más probabilidades de experimentar enfermedad depresiva (4-6). La depresión afecta a cerca de una quinta parte de los pacientes con infarto de miocardio (IM) y está asociada con un riesgo (dos o más veces) mayor de mortalidad por cualquier causa, mortalidad cardiovascular o nuevos eventos cardiovasculares (7). Sin embargo, pruebas aleatorias de tratamiento de la depresión no han demostrado que al tratar esta, mejore el pronóstico de la enfermedad cardiaca post-IM $(8,9)$.

Hasta la fecha, la asociación de enfermedad cardiaca con otros trastornos mentales comunes, en especial los trastornos de ansiedad y de abuso/dependencia de alcohol, han recibido menos atención. Si bien se han realizado investigaciones acerca de la co-morbilidad enfermedad cardiaca-trastorno mental en países desarrollados, no es clara aun la existencia de diferencias en las prevalencias de dichos trastornos en individuos con y sin enfermedad cardiaca (10). Consecuentemente es poco lo conocido con respecto a su asociación en países en vías de desarrollo como Colombia. Dado el dramático incremento en la expectativa de vida que ha ocurrido en las últimas décadas en el país, es necesario mejorar la información acerca de la co-ocurrencia de enfermedad cardiaca y trastornos mentales.

Utilizando datos del Estudio Nacional de Salud Mental, Colombia 2003, que hace parte de la Encuesta Mundial de Salud Mental de la Organización Mundial de la Salud (OMS), presentamos información novedosa en relación con la ocurrencia de trastornos mentales comunes entre personas que reportan enfermedad cardiaca. Los objetivos de este artículo son: 1) presentar la prevalencia estimada de trastornos del estado de ánimo, ansiedad y por consumo de alcohol en personas con enfermedad cardiaca en Colombia; 2) identificar los trastornos mentales específicos más fuertemente asociados con enfermedad cardiaca. Este artículo ofrece la primera información sobre la ocurrencia de trastornos del estado de ánimo, de ansiedad y por consumo de alcohol entre personas con enfermedad cardiaca en una muestra de población general. Este da una perspectiva general sobre 
las asociaciones de trastornos comunes de estado de ánimo, ansiedad y por consumo de alcohol con enfermedad cardiaca en Colombia.

\section{Métodos}

Muestra. Una encuesta en población general fue realizada en todo el país, mediante un diseño probabilístico, multietápico y estratificado de hogares a nivel nacional. Todas las entrevistas fueron llevadas a cabo cara a cara por encuestadores legos previamente entrenados. Fueron utilizadas sub-muestras al interior de la encuesta para reducir la carga en el entrevistado dividiendo la entrevista en dos partes. La parte 1 incluyó la evaluación diagnóstica de los principales trastornos mentales. La parte 2 incluyó información adicional relevante para un amplio rango de objetivos de la encuesta, entre ellos la evaluación de enfermedades físicas crónicas. Todos los encuestados completaron la parte 1. Quines reunieron criterios para cualquier trastorno mental y una muestra probabilística en los que no ocurrió esto, se les realizó la parte 2. Los resultados de los encuestados de la parte 2 fueron ponderados por el inverso de la probabilidad final de selección para la parte 2 de la encuesta, para garantizar la representatividad poblacional de los resultados. Los análisis en este artículo fueron basados en la parte 2 ponderada de la muestra. Ponderaciones adicionales fueron usadas para ajustar por probabilidades diferenciales de selección dentro de los hogares y por la distribución socio-demográfica de la población.

Entrenamiento y trabajo de campo: el equipo central de la World Mental Health (WMH) entrenó supervisores bilingües para Colombia. Se utilizaron los documentos y procedimientos pertinentes para el entrenamiento de los encuestadores y se siguió el protocolo de traducción de la OMS para traducir los instrumentos y el material de entrenamiento. La información sobre los objetivos y procedimientos del estudio, el uso y protección de los datos y los derechos de las personas encuestadas fueron entregadas tanto en forma verbal como escrita a aquellas potencialmente elegibles antes de obtener el consentimiento informado verbal para participar en la encuesta. Los protocolos de control de calidad, descritos con mayor detalle en otra publicación (11), fueron estandarizados con los de la Encuesta Mundial de Salud Mental para asegurar la exactitud del entrevistador y para definir la limpieza de datos y los procedimientos de codificación. El Comité de Ética de la Fundación FES Social aprobó y monitorizó el acatamiento de los procedimientos para obtener el consentimiento informado y los derechos de las personas.

Estado de trastorno mental: la encuesta utilizó la WHO Composite International Diagnostic Interview $(12,13)$, versión de la Encuesta Mundial de Salud Mental, para evaluar trastornos. Los diagnósticos considerados en este artículo incluyen los trastornos de ansiedad (trastorno de ansiedad generalizada, trastorno de angustia y/o agorafobia, trastorno por estrés post traumático y fobia social), trastornos del estado de ánimo (distimia y episodio depresivo mayor) y abuso/dependencia de alcohol. Los trastornos fueron evaluados utilizando las definiciones y criterios del Manual Diagnóstico y Estadístico de Trastornos Mentales, Cuarta Edición (DSM-IV) American Psychiatric Association, 1994 (14). Se utilizaron los criterios de exclusión de organicidad del CIDI para realizar todos los diagnósticos. Las pruebas de campo y posteriores estudios de calibración clínica mostraron que los trastornos considerados en este estudio fueron evaluados con aceptable confiabilidad (12).

Estado de enfermedad cardiaca: con una serie de preguntas acerca de enfermedades físicas crónicas, adaptadas de la U.S Health Interview Survey (National Center for Health Statistics, 1994) (15), los encuestados fueron interrogados acerca de la presencia de condiciones crónicas. Se les preguntó si un médico u otro profesional de la salud alguna vez le había dicho que tenía una enfermedad del corazón. Estudios previos en países desarrollados sugieren que el auto reporte de enfermedades graves como enfermedad cardiaca y diabetes tienen una validez aceptable $(15,16)$. 
T abla 1.Prevalencia (\%) de T rastornos del estado deánimo entre personas con enfermedad cardiaca vs. sin enfermedad cardiaca.

\begin{tabular}{llllll}
\multicolumn{2}{c}{ Depresión Mayor } & \multicolumn{2}{c}{ Distimia } \\
No & & & No & \\
Enfermedad & Enfermedad & & Enfermedad & Enfermedad \\
Cardiaca & Cardiaca & OR $(\mathrm{Cl})$ & Cardiaca & Cardiaca & OR $(\mathrm{Cl})$ \\
5.9 & 13.9 & $2.6(1.5,4.8)^{*}$ & 0.9 & 5.0 & $5.6(1.8,17.4)^{*}$ \\
\hline
\end{tabular}

Kriegsman y colaboradores en 1996 reportaron un aceptable nivel de concordancia entre los resultados obtenidos a partir de las historias clínicas de los médicos generales y los auto reportes de enfermedad del corazón basados en entrevistas (kappa de 0.64) para enfermedad cardiaca isquémica, similar a los valores para diabetes (0.74) y asma (0.58). Estudios previos reportaron niveles similares de concordancia para infarto de miocardio y enfermedad cardiaca coronaria $(17,18)$.

Métodos de análisis: este artículo reporta tasas de prevalencia para trastornos mentales específicos en personas con y sin enfermedad cardiaca. Para esta encuesta, fueron calculados usando los Odds ratio para la valoración del nivel de asociación entre la ocurrencia de cada trastorno mental y enfermedad cardiaca. Dichos valores fueron ajustados por edad y sexo. Teniendo en cuenta que los trastornos del estado de ánimo disminuyen su prevalencia con la edad y tienden a ser más comunes en mujeres y que la enfermedad cardiaca incrementa su prevalencia con la edad y tiende a ser más frecuente en hombres, es esencial controlar el efecto confusor de las variables edad y sexo en la evaluación de la asociación de enfermedad cardiaca y trastornos del estado de ánimo. Por otra parte, fueron calculados intervalos de confianza al 95\% utilizando el método de linealización de Taylor (19) con software SUDAAN (20) para ajustar por el efecto de diseño (conglomeración y auto-ponderación).

\section{Resultados}

Características de la muestra: el tamaño de la muestra de la encuesta fue de 4426 individuos. La tasa de respuesta observada fue de $88.7 \%$. La proporción de personas de 60 o más años fue de $5.3 \%$ y el porcentaje de quienes habían completado educación secundaria fue de $46.4 \%$. La enfermedad cardiaca auto reportada fue común, con una prevalencia cercana al 3\%. La prevalencia fue mayor entre personas mayores, especialmente aquellas que no completaron la educación secundaria y en general entre hombres (datos no mostrados).

Trastornos del estado de ánimo y enfermedad cardiaca: la depresión mayor fue característicamente común en personas con enfermedad cardiaca, datos que se muestran en la Tabla 1. La prevalencia de depresión mayor en personas con enfermedad cardiaca fue de 13.9 $\%$ (CI). La prevalencia de distimia fue inferior significativamente. La comparación de la prevalencia de depresión mayor y distimia entre personas con enfermedad cardiaca versus personas sin enfermedad cardiaca mostraron diferencias significativas.

Como se muestra en la Tabla 1 , los Odds ratio ajustados fueron significativamente mayores que uno (indicando un efecto de incremento en el riesgo....). También indican una asociación significativa entre el riesgo de padecer enfermedad cardiaca y distimia.

Trastornos de ansiedad y enfermedad cardiaca: a lo largo de la encuesta, los trastornos específicos de ansiedad (trastorno de ansiedad generalizada, trastorno de angustia/agorafobia, fobia social y trastorno por estrés post traumático (TEP) fueron generalmente menos prevalentes que la depresión mayor. Entre las personas con enfermedad cardiaca, la prevalencia de trastorno de ansiedad generalizada (TAG) alcanzó 1.5 (CI), la fobia social alcanzó 4.8 (CI) y el trastorno de angustia/ 
T abla 2. Prevalencia (\%) de trastornos de ansiedad entre Las personas con enfermedad cardiaca vs. sin enfermedad cardiaca.

\begin{tabular}{lcccc} 
& \multicolumn{2}{c}{ TRANSTORNOS DEANSIEDAD } & \\
& $\begin{array}{c}\text { Ansiedad } \\
\text { Generalizada }\end{array}$ & $\begin{array}{c}\text { Agorafobia o Trastorno } \\
\text { de Angustia }\end{array}$ & Fobia Social & Trastorno por estrés \\
No Enfermedad Cardiaca & 1.0 & 2.1 & 2.8 & post traumático \\
Enfermedad Cardiaca & 1.5 & 4.9 & 4.8 & 0.6 \\
OR $(\mathrm{Cl})$ & $1.6(0.4,6.3)$ & $2.5(1.0,6.3)$ & $2.1(0.8,5.1)$ & 0.5 \\
\hline
\end{tabular}

agorafobia llegó a 4.9 (CI). El TEP fue relativamente poco común entre personas con enfermedad cardiaca. Estos datos se muestran en la Tabla 2.

A excepción del TEP en los trastornos de ansiedad los Odds ratio fueron significativamente mayores que uno. La asociación de cualquier trastorno de ansiedad con enfermedad cardiaca muestra un patrón similar al observado para cualquier trastorno del estado de ánimo. El Odds ratio estimado fue $2.2(1.9,2.5)$. El test de heterogeneidad para los Odds ratios de todos los trastornos de ansiedad fue estadísticamente significativo en todos los casos $(\mathrm{p}=0.01)$. Estos resultados indican que la fortaleza de la asociación de trastornos de ansiedad, como una clase, con enfermedad cardiaca es comparable a la observada para los trastornos del estado de ánimo - un Odds ratio de cerca de 2 de trastornos de ansiedad para personas con enfermedad cardiaca versus personas sin enfermedad cardiaca-. Sin embargo, la variación en los estimados de la fuerza de la asociación entre trastorno de ansiedad y enfermedad cardiaca fue mayor que los observados para trastornos del estado de ánimo, posiblemente debido a la baja prevalencia de estos trastornos.

Trastornos por uso de alcohol y enfermedad cardiaca: como se muestra en la Tabla 3, la prevalencia de abuso o dependencia de alcohol llega a 3.1. Los Odds ratio para la asociación de abuso o dependencia de alcohol con enfermedad cardiaca fueron estimados. Estos arrojaron mayores que 1.0, sin embargo, los intervalos de confianza correspondientes incluyeron el valor nulo. Dado que la prevalencia del uso de alcohol disminuye marcadamente con la edad y es inferior en mujeres, en tanto que la prevalencia de enfermedad cardiaca se incrementa con la edad y es generalmente mayor entre hombres, el ajuste por edad y sexo es importante para reducir el efecto de esta situación al evaluar dicha asociación. El estimado de odds ratio para la asociación de abuso o dependencia de alcohol y enfermedad cardiaca fue de 1.4 (1.0 a 1.9). Estos resultados sugieren que el abuso de alcohol puede ocurrir con mayor frecuencia entre personas con enfermedad cardiaca, aunque en general los resultados no sugieren una fuerte asociación.

\section{Discusión}

Este informe ofrece una evaluación de la frecuencia y asociación tanto de los trastornos del estado de ánimo y de ansiedad con enfermedad cardiaca basada en la primera población nacional en gran escala. Dos hallazgos claves emergen del presente estudio. Primero, personas con enfermedad cardiaca tuvieron mayor probabilidad de experimentar trastornos de ansiedad o del estado de ánimo que personas comparable sin enfermedad cardiaca después de tener en cuenta diferencias por edad y sexo. Segundo, los trastornos individuales del estado de ánimo y de ansiedad tienen una asociación similar con enfermedad cardiaca. Estos hallazgos tienen implicaciones para la práctica clínica, sugieren que los clínicos necesitan ser concientes del espectro de trastornos del estado de ánimo y de ansiedad que ocurre con mayor frecuencia entre personas con enfermad cardiaca en vez de enfocarse solamente en un trastorno 
T abla 3.Prevalencia (\%) de abuso/dependencia de alcohol entre personas con vs. sin enfermedad cardiaca.

\begin{tabular}{ccc}
\multicolumn{3}{c}{ Abuso/dependencia de alcohol } \\
No Enfermedad Cardiaca & Enfermedad Cardiaca & OR (Cl) \\
& 3.1 & $1.5(0.3,7.3)$ \\
\hline
\end{tabular}

específico (por ejemplo depresión mayor).

Las asociaciones observadas tienen limitadas implicaciones etiológicas; el diseño utilizado (corte transversal) no permite inferir respecto a la temporalidad de ocurrencia entre enfermedad cardiaca y los trastornos de estado de ánimo y de ansiedad. Sin embargo, el hecho de que los trastornos del estado de ánimo y de ansiedad muestran un nivel similar de asociación con la enfermedad cardiaca sugiere que cualquier efecto de la enfermedad cardiaca sobre el funcionamiento psicológico puede influir en los estados de ansiedad así como los trastornos del estado de ánimo. De igual manera, si el trastorno psicológico incrementa los riesgos de enfermedad cardiaca, el papel de la ansiedad debe ser considerado conjuntamente con la depresión. Los resultados del abuso y la dependencia al alcohol sugieren que el abuso de este puede ser más común entre personas con enfermedad cardiaca que aquellos sin esta.

Limitaciones: la limitación más notable de esta investigación es la determinación de enfermedad cardiaca basada en auto reporte. Dado que el estudio Nacional de Salud Mental, Colombia 2003 fue llevado a cabo en una gran población en toda Colombia, no era posible acceder a historias clínicas o llevar a cabo una evaluación medica estandarizada para determinar si la enfermedad cardiaca estaba presente o ausente. Investigaciones previas en países desarrollados sugieren que la enfermedad cardiaca auto reportada tiene aceptable validez con un kappa que excede generalmente 0.60 (1618). Otra limitación es que la encuesta $\mathrm{WMH}$ evalúa solo la presencia de la enfermedad cardiaca y no su severidad o la naturaleza exacta de la enfermedad cardiaca reportada. La enfermedad cardiaca es generalmente sub reportada (16). No hay evidencia que personas con trastornos psicológicos tengan mayor probabilidad de reportar menos la presencia de enfermedad crónica encontraron que la «afectividad negativa» (un rasgo relacionado con trastorno del estado de animo) fue asociado con reportes incrementados de síntomas y enfermedades crónicas (21).

Sin embargo, es posible que la asociación de trastornos del estado de ánimo y ansiedad con enfermedad cardiaca sea mejor explicado por factores inespecíficos que influyen el reporte de enfermedad que con la asociación de enfermedad cardiaca específica. Las personas ansiosas y depresivas pueden ser más sensibles y menos olvidadizas al malestar físico con respecto a diagnósticos previos de problemas físicos. Dado que los trastornos del estado de ánimo y de ansiedad están asociados con muchas condiciones físicas crónicas, es necesaria mayor investigación para entender los factores que pueden influir la asociación de trastornos del estado de animo y ansiedad con diversas enfermedades crónicas (5).

La prevalencia de enfermedad cardiaca en el Estudio Nacional de Salud Mental es coincidente con los patrones epidemiológicos (p. e. incremento de la prevalencia con la edad; generalmente mayor prevalencia en hombres). Es de anotar que el Estudio Nacional de Salud Mental, Colombia 2003 incluyó el uso de métodos estandarizados y bien validados para diagnosticar trastornos mentales en encuestas poblacionales y además el tamaño y diversidad de la población encuestada. Por lo tanto, fue posible desarrollar estimativos basados en poblaciones con un rango de trastornos mentales sin precedentes entre personas en hogares, no institucionalizadas, que reportan enfermedad cardiaca.

Los resultados de este estudio permiten anotar, a manera de conclusión, que los trastornos del estado de ánimo y de ansiedad ocurren con mayor frecuencia en personas con enfermedad cardiaca. El abuso de alcohol 
fue asociado con trastornos mentales y con enfermedad cardiaca. Dada la asociación de trastornos del estado del ánimo y ansiedad con discapacidad funcional (3), estos resultados sugieren que los clínicos deben ser concientes de la mayor ocurrencia de trastornos del estado de ánimo y de ansiedad entre pacientes con enfermedad cardiaca. Esta investigación no da luces si los trastornos psicológicos son causa o consecuencia de enfermedad cardiaca, pero sugiere que los esfuerzos para entender las relaciones causales entre enfermedad cardiaca y enfermedad psicológica deben considerar los trastornos de ansiedad así como los del estado de ánimo.

\section{Agradecimientos}

El estudio Nacional de Salud Mental, Colombia 2003 (ENSM) fue financiado por el Ministerio de la Protección Social y hace parte de la Encuesta Mundial de Salud Mental (WMH) de la Organización Mundial de la Salud. Agradecemos al equipo de la WMH por el apoyo con los instrumentos y análisis de datos. Estas actividades fueron apoyadas por el Instituto Nacional de Salud Mental de los Estados Unidos (R01MH070884), la Fundación John D. and Catherine T. MacArthur, la Fundación Pfizer, el Servicio de Salud Pública de los Estados Unidos (R13-MH066849, R01-MH069864, y R01 DA016558), el Centro Internacional Fogarty (FIRCA R01-TW006481), la Organización Panamericana de la Salud, Eli Lilly y Compañía, Ortho-McNeil Pharmaceutical, Inc., GlaxoSmithKline, y Bristol-Myers Squibb. Una lista completa de las publicaciones de la WMH puede ser encontrada en http:// www.hcp.med.harvard.edu/wmh/. NOV

\section{Referencias}

1. Murray C, Lopez A. Alternative projections of mortality and disability by cause 1990-2020. Global Burden of Disease Study. Lancet 1997;349:1498-1504.

2. Insel TR, Charney DS. Research on Major Depression. Strategies and priorities. JAMA 2003;289:3167-3168.

3. Ormel J, VonKorff M, Ustun TB, Pini S, Korten A, Oldehinkel AJ. Common mental disorders and disability across cultures.
Results from the WHO Collaborative Primary Care Study. JAMA 1994;272:1741-1748.

4. Penninx BW, Beekman AT, Honig A, Deeg, DJ, Schoevers RA, van Eijk JT, van Tilburg W. Depression and cardiac mortality: results from a community-based longitudinal study. Arch Gen Psychiatry 2001;58:221-227.

5. Evans DL, Charney DS, Lewis L, Golden RN, Gorman JM, Rama Krishnan KR, et al. Mood disorders in the medically ill: Scientific Review and Recommendations. Biol Psychiatry 2005;58:175-189.

6. Rudisch B, Nemeroff CB. Epidemiology of comorbid coronary artery disease and depression. Biol Psychiatry 2003;54:227240 .

7. Van Melle JP, de Jonge P, Spijkerman TA, Tijssen JG, Ormel $J$, van Veldhuisen DJ et al. Prognostic association of depression following myocardial infarction with mortality and cardiovascular events: a meta-analysis. Psychosom Med 2004;66:814-822.

8. Berkman LF, Blumenthal J, Burg M, Carney RM, Catellier $\mathrm{D}$, Cowan MJ et al. Effects of treating depression and lowperceived social support on clinical events after myocardial infarction - The enhancing recovery in coronary heart disease patients (ENRICHD) randomized trial. JAMA 2003;289:3106-3116.

9. Glassman AH, O'Connor CM, Califf RM, Swedberg K, Schwartz P, Bigger JT, Jr. et al. Sertraline treatment of major depression in patients with acute MI or unstable angina. JAMA 2002;288:701-709.

10. Baune BT, Adrian I, Arolt V, Berger K. Associations between major depression, bipolar disorders, dysthymia and cardiovascular diseases in the general adult population. Psychother Psychosom. 2006;75:319-326.

11. Kessler R, Berglund P, Chiu WT, Demler O, Heeringa S, Hiripi E, Jin R, Pennell B-P, Walters EE, Zaslavsky A, Zheng H. The US National Comorbidity Survey Replication (NCS-R): design and field procedures. Int $\mathrm{J}$ Methods Psychiatr Res. 2004;13:69-92.

12. Wittchen HU. Reliability and validity studies of the WHO Composite International Diagnostic Interview (CIDI): a critical review. J Psychiatr Res. 1994;28:57-84.

13. Kessler RC, Ustun TB. The World Mental Health (WMH) Survey Initiative Version of the World Health Organization (WHO) Composite International Diagnostic Interview (CIDI). Int J Methods Psychiatr Res. 2004;13: 93-121.

14. American Psychiatric Association. Diagnostic and Statistical Manual of Mental Disorders, Fourth ed. Washington, DC: American Psychiatric Association; 1994.

15. National Center for Health Statistics. Evaluation of National Health Interview Survey diagnostic reporting. Vital and Health Statistics 2 1994;120:1-116.

16. Kriegsman DM, Penninx BW, van Eijk JT, Boeke AJ, Deeg DJ. Self-reports and general practioner information on the presence of chronic diseases in communiting dwelling elderly. J Clin Epidemiol. 1996;12:1407-1417. 
17. Kehoe R, Wu S-Y, Leske MC, Chylack LT. Comparing selfreported and physician-reported medical history. Am J Epidemiol 1994;139:813-818

18. Tretli S, Lund-Larsen PG, Foss, OP. Reliability of questionnaire information on cardiovascular disease and diabetes: cardiovascular disease study in Finnmark county. J Epidemiol Comm Health 1982;36:269-273.
19. Wolter KM. Introduction to Variance Estimation. New York, NY: Springer-Verlag; 1985.

20. SUDAAN [computer program] Version 8.0.1. Research Triangle Park, NC: Research Triange Institute; 2002.

21. Kolk AM, Hanewald GJ, Schagen S, Gijsbers van Wijk CM. Predicting medically unexplained physical symptoms and health care utilization. A symptom-perception approach. J Psychosom Res 2002;52:35-44.
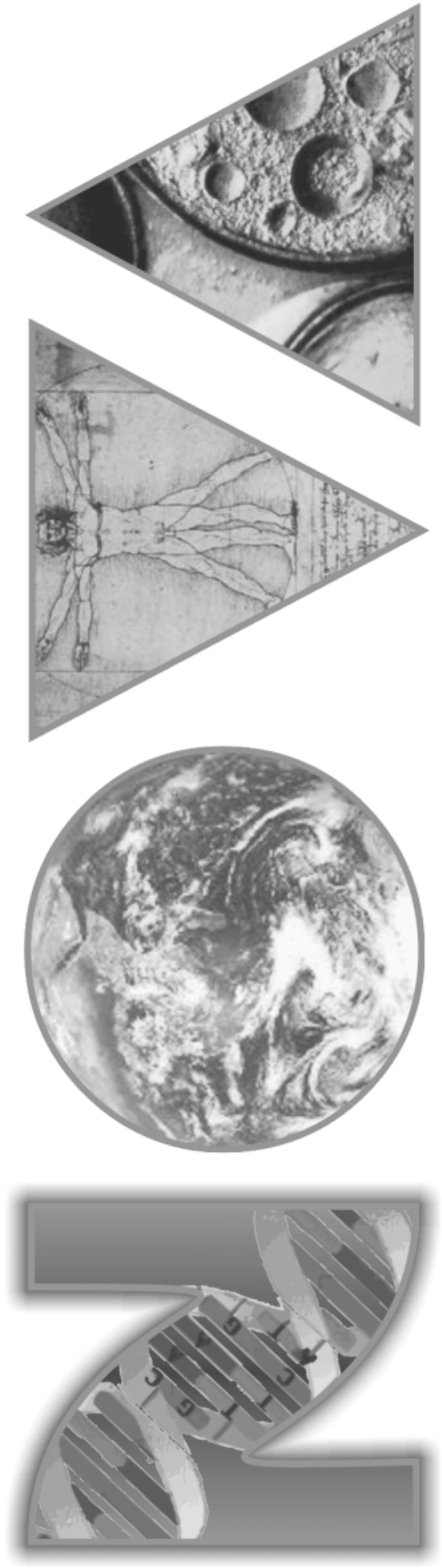